\title{
LA PUERTA DE LA MISERICORDIA
}

\section{Introducción}

La ciudad de Jerusalén es una ciudad amurallada. Por lo tanto, para entrar y salir son necesarias puertas. La llamada actualmente «ciudad antigua de Jerusalén» corresponde a la parte de la ciudad que ha quedado dentro de las murallas. Pero estas murallas actuales no son antiguas -si tenemos en cuenta la milenaria historia de Jerusalén-, sino del siglo XVI, época en que dominaba Imperio otomano. Fue el sultán Solimán el Magnífico (1520-1566) quien reconstruyó las murallas, en varios lugares sobre los restos de las antiguas. Dicha reconstrucción duró de 1535 a 1538.

Asimismo, las puertas de la ciudad amurallada pertenecen a este período. Y también varias de ellas se ubican en los lugares de sus respectivas antiguas puertas -incluso en algunos casos conservando el antiguo nombre-.

La actual muralla cuenta con ocho puertas. Siete están abiertas y son perfectamente utilizables. La octava ${ }^{1}$ está sellada y permanece así -con algunos breves períodos de apertura que desarrollaremos en el presente escrito- desde el año 810, poco después de la conquista musulmana de Jerusalén.

La rica historia de la puerta hace que tenga varios nombres según las épocas y las distintas tradiciones religiosas: puerta de la Misericordia, puerta Shushan, puerta Este u Oriental. Nosotros la denominaremos «puerta Este» cuando nos refiramos en forma genérica, y cuando estemos tratando textos que le dan otro nombre más específico, lo usaremos.

En el presente trabajo nos proponemos hacer un breve recorrido histórico, centrándonos en las fuentes escritas -bíblicas, extrabíblicas históri-

1 Estrictamente, deberíamos hablar de más puertas selladas. Restos también de la reforma y ampliación hecha por Herodes, como por ejemplo la puerta de Barclay, en el lado oeste (exactamente en el actual Kotel), la puerta doble y la puerta triple, hacia el lado sur (hacia la colina del Ofel y la Ciudad de David). 
cas y misnaicas- y en los descubrimientos e interpretaciones de los restos arqueológicos.

\section{Recorrido histórico de la puerta Este}

\subsection{Período del Primer Templo}

Es muy poco lo que podemos rastrear arqueológicamente de este período debido a la distancia en el tiempo, a las numerosas destrucciones y reconstrucciones y a la situación político-religiosa, que impide cualquier tipo de excavación arqueológica. Solo disponemos de fuentes escritas. En 1 Re 6-7 se relata la construcción del Templo en sí, pero no se mencionan puertas en la muralla este. Igualmente sucede con el relato de $2 \mathrm{Cr} 3-4$. En $2 \mathrm{Cr}$ 4,22 se mencionan dos puertas recubiertas en oro, la del lugar Santísimo y la del Templo. Las puertas que se mencionan en 2 Re 11,6.19 no son claramente identificables y nos resultan desconocidas. Asimismo la «puerta superior» en $2 \operatorname{Re} 15,35$ y 2 Cr 27, 10.

\subsection{Período del Segundo Templo}

Por consenso llamamos Segundo Templo al tiempo que va desde la reconstrucción completada en el año 515 a. C., luego de la liberación de la cautividad babilónica y gracias al auspicio de Ciro, rey de Persia, pasando por la ampliación del Gran Templo realizado por Herodes el Grande, comenzada en el año 20 a. C., hasta la destrucción en el año 70 en manos de la Legión X Fretensis, comandada por el general Tito (luego emperador).

Nehemías llegó a Jerusalén en el año 445 a. C. y fue el gran reconstructor de la ciudad. El libro de Nehemías describe el recorrido nocturno que realizó para conocer el estado de destrucción de las fortificaciones y murallas. Sobre la base del texto bíblico se puede conocer por dónde pasaba, ya sea aproximada o exactamente, la segunda muralla -también llamada la «muralla ancha»-. Algunas de ellas fueron identificadas arqueológicamente con exactitud: la puerta del Valle, la puerta de los Desperdicios, la puerta de la Fuente, la muralla de la piscina de Siloé y la sección escalonada de la Ciudad de David ${ }^{2}$.

2 D. BAнAт, The Illustrated Atlas of Jerusalem. Carta Jerusalem. Jerusalén, 1996, p. 34. 
Otro grupo de restos arqueológicos mencionados por Nehemías que han sido identificados se encuentran por el lado norte del monte del Templo: la puerta de las Ovejas, la torre Meá («cien» en hebreo: es la «torre de los cien») y la torre de Hananel. Aunque la localización exacta es discutida por algunos arqueólogos ${ }^{3}$.

Lamentablemente para el propósito de nuestro escrito, no se sabe con exactitud por dónde pasaba la muralla este. El arqueólogo israelí Dan Bahat menciona que las estructuras encontradas y analizadas por la famosa arqueóloga británica Kathleen Kenyon, que ella creía que eran de la muralla de la época que estamos mencionando, son restos de construcciones que evidentemente estaban dentro de la muralla, aún no descubierta ${ }^{4}$. Las fuentes escritas con que contamos ${ }^{5}$ que describen la ciudad de Jerusalén y el monte del Templo desde la vuelta del destierro hasta la ampliación realizada por Herodes no mencionan la muralla este y la puerta Este. En el «recorrido nocturno» de Neh 2,11-15 se describe que sube por el torrente -Cedrón-, inspecciona la muralla, pero vuelve a la puerta del Valle, sin mencionar ninguna puerta del lado este. Cuando habla de «valle» debemos evitar la tentación moderna -debido a los cambios topográficos- de identificarla con el valle Cedrón. La puerta del Valle a la que se refiere en dicho período es la que conduce al valle Tyropeón, que es el valle central que atraviesa Jerusalén de norte a sur -actualmente desde la puerta de Damasco hasta la puerta de los Desperdicios, recorriendo la calle El-Wad ${ }^{6}$, separando el monte del Templo del barrio alto residencial.

En el capítulo 3 del libro de Nehemías se relata la reconstrucción de las puertas y murallas. Este es el capítulo más interesante para nuestro estudio. Comienza con las puertas del lado norte más arriba mencionadas: la puerta de las Ovejas, la torre Meá y la torre de Hananel. Transcribiremos el primer versículo debido a una serie de detalles importantes: «Entonces se levantó Eliashib, el sumo sacerdote, con sus hermanos, los sacerdotes, y reconstruyeron la puerta de las Ovejas: la consagraron y colocaron sus hojas; luego continuaron hasta la torre de los Cien y hasta la torre de Jananel, y consagraron la muralla». Dos veces se menciona la consagración/santificación -en ambas se utiliza en verbo קרש-, primero de la puerta y luego de toda la muralla. Siguiendo el recorrido hacia el sur sobre la muralla oeste

3 Ibid., p. 36.

${ }^{4}$ Ibid.

${ }^{5}$ Además del libro de Nehemías, mencionado anteriormente, tenemos la Carta de Aristeas, escrita en el siglo ॥ a. C.

6 Justamente, wad y wadi significan en árabe «valle». 
se menciona la puerta de los Pescados y la puerta Vieja hasta el muro Ancho; de allí, siempre siguiendo hacia el sur, la torre de los Hornos, la puerta del Valle y la puerta de la Basura. Llegamos a la puerta de la Fuente, que, al ser mencionada junto al «estanque del canal hasta las escaleras que bajan a la Ciudad de David», podemos deducir que se trata del extremo sur de la Ciudad de David, y desde allí nuevamente comienza a dirigirse hacia el norte, esta vez por el lado este. Se menciona -ya en el v. 26- la puerta de las Aguas, que da hacia el este. Luego se menciona el Ofel y la puerta de los Caballos - סו:סים-, por lo que podemos deducir que estamos al sur del monte del Templo, en la colina que separa al mismo de la Ciudad de David. Inmediatamente aparece la puerta Oriental -מזירָּחי-. Luego la puerta de la

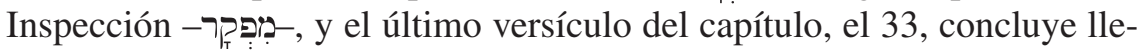
gando nuevamente a la puerta de las Ovejas.

Del período asmoneo -inaugurado con la revuelta asmonea en el año 167 a. C.- contamos como fuente principal los libros bíblicos de los Macabeos. Existió una expansión de la ciudad hacia el oeste, pero en cuanto al monte del Templo y la muralla este con su puerta no hay noticias, excepto en la zona de la Ciudad de David, cuyas fortificaciones macabeas fueron descubiertas y estudiadas en 1923 por Macalister y Duncan, y posteriormente Kenyon. El asmoneo Jonatán comenzó ${ }^{7}$ la construcción de murallas, tarea que será completada por su hermano Simón, pero en la zona del monte Sión.

No disponemos de fuentes que describan las construcciones en el monte del Templo durante el período asmoneo. Pero el análisis de los restos arqueológicos y la comparación con textos de Flavio Josefo, sumadas a las descripciones de la Misná, nos aportan datos acerca de los numerosos cambios que efectivamente hubo. Detengámonos en este último texto, ya que aquí aparece mencionada la puerta Este ${ }^{8}$.

El tratado Middot $^{9}$ de la Misná menciona cinco puertas que facilitan el acceso al monte del Templo: al norte, la puerta Tadi; al oeste, la puerta Kinopus; al sur, las puertas Huldá ${ }^{10}$, y, finalmente, al este, la puerta Shushan.

El texto citado de la Misná especifica con una importante aclaración

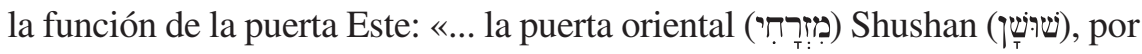

$71 \mathrm{Mac} 10,10$.

${ }^{8}$ Vale recordar que, si bien la Misná fue redactada y compilada hacia el siglo ॥ d. C., algunos estudiosos creen que la descripción en particular de las puertas y murallas del monte del Templo del tratado Middot se remontan al período asmoneo. Cf. D. Bанат, The Illustrated Atlas of Jerusalem, p. 39.

9 Middot 1,3.

10 Posteriormente serán llamadas puerta Doble y puerta Triple, cuyos restos -tapiados- son visibles hasta el día de hoy. 
la cual el sacerdote que quema la vaca, y la vaca, y todos sus asistentes, sa-

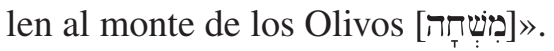

Algunas particularidades lingüísticas para destacar:

מִזיר designa el lugar desde donde sale el sol. De ahí la forma adje-

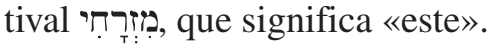

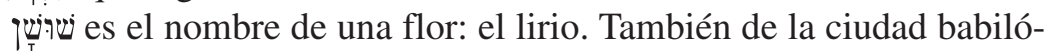
nica de Susa.

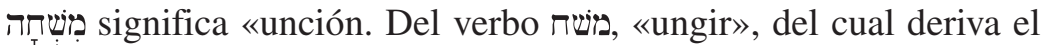

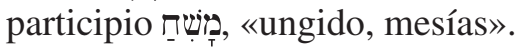

Acerca del origen del nombre de esta puerta, el texto citado de la Misná, Middot 1,3 explica que se llamaba así porque sobre la puerta había una imagen -posiblemente un relieve ${ }^{11}$ - del templo de Susa, en Persia. Podría ser porque de allí venían los retornados del exilio, gracias a Ciro, justamente el rey de Persia. De hecho, numerosos textos del Antiguo Testamento alaban a Ciro por haber posibilitado el retorno a la tierra prometida y haber permitido y contribuido a la reconstrucción del Templo. Por ejemplo, Esd 1,1 menciona que Ciro tenía el «espíritu del Señor», e Is 45,1 lo llama «mesías».

Sin embargo, en este trabajo nos animamos a hacer una propuesta que no pretende ser alternativa a la tradición misnaica, pero que resulta sumamente sugestiva. Podemos ver que en 1 Re 7,19.22 se describe que los capiteles que estaban encima de las columnas en el vestíbulo del Templo

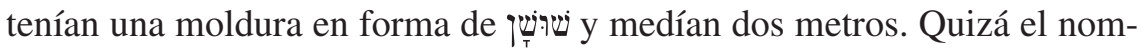
bre de la puerta Este se llamaba así porque desde el este -especialmente desde el monte de los Olivos $^{12}$ - se destacaban con claridad esas molduras. Si bien el texto del libro de los Reyes habla del primer Templo, el de Salomón, podría ser un recuerdo que quedó en la memoria judía o una impostación realizada por la Misná en honor a aquel primer Templo. En cualquier caso parece clara la relación mencionada.

En el año 67 a. C., Pompeyo sitia y conquista Jerusalén. Comienza así el período de dominación del Imperio romano.

Del último período en cuanto a las construcciones y ampliaciones del segundo Templo, llamado «herodiano» ${ }^{13}$, tenemos una gran cantidad de

11 Así lo interpreta Rose Guide to Temple. Bristol Works, 2012, p. 75 (Accordance edition hypertexted and formatted by OakTree Software, Inc. Version 1.2).

12 La Misná, Middot 3,4, dice que los muros interiores que rodeaban el Templo por el lado este no eran altos como los otros, de manera tal que el sacerdote que quemaba la vaca roja sobre el monte de los Olivos pudiera ver la puerta del lugar Santísimo cuando era rociada con la sangre del sacrificio.

13 Herodes el Grande (73 a. C. - 4 a. C.) fue nombrado procurador de Judea en el año 37 a. C. por el emperador Julio César. En el año 40 a. C. consiguió el título de 
información por las fuentes, especialmente Flavio Josefo, y numerosos restos que han llegado hasta el día de hoy. Sus obras no se limitan al monte del Templo, sino que se expanden por toda la ciudad de Jerusalén. No es nuestro objetivo describir ni estudiar estas obras; simplemente mencionemos la ampliación y terminación de la primer muralla -que había comenzado en el período asmoneo-, especialmente al sur de la ciudadela para proteger la ciudad alta, la adición de una segunda muralla hacia el norte, más allá de la ciudad alta, numerosas torres, etc. Gran parte de estas murallas resultaron tan sólidas que las actuales otomanas están construidas en muchos sitios sobre aquellas herodianas. Asimismo se agregaron puertas, como la puerta de los Esenios, la puerta Oculta ${ }^{14}$, la puerta Gennath ${ }^{15}$, la puerta de Warren, la puerta de Barclay; puentes para cruzar el valle entre la ciudad y el monte del Templo $^{16}$-cuyos restos son conocidos hoy día como arco de Wilson y arco de Robinson, y una fortaleza adyacente en el lado norte del monte del Templo llamada «Antonia». Solo mencionamos la ampliación y construcciones de un intrincado sistema de acueductos y piscinas para proporcionar mucha agua al Templo, debido a la cantidad de sacrificios que se realizaban ${ }^{17}$.

Centrándonos en nuestro objetivo del lado este de la muralla, encontramos restos de otra antigua puerta. Se trata de la esquina sur de la muralla este, tradicionalmente conocida como el «pináculo del Templo». Charles Warren realizó un estudio con minuciosos planos en 1884, en el que nota un arco de siete metros, y sobre él restos de una puerta doble desde la cual descendían escaleras que se dirigían al pie de dicha muralla herodiana ${ }^{18}$. Por allí corría una calle que se dirigía hacia el norte, hacia la puerta Este.

rey, otorgado por el emperador Marco Antonio. Como mencionamos más arriba, Herodes comenzó la ampliación y embellecimiento del Templo y sus instalaciones, incluyendo una gigantesca explanada para soportar todo, en el año 20 a. C.

${ }^{14}$ Cf. Flavio Josefo, Guerra de los judíos V.6.5, en The Works of Flavius Josephus, Complete and Unabridged. New Updated Edition. Translated by W. WHISTON. Peabody, MA, Hendrickson, 1987 (Electronic text hypertexted and prepared by OakTree Software, Inc. Version 1.8).

15 Muy posiblemente la puerta que conducía al «jardín» mencionado en Jn 19,41 en la colina del Gólgota.

16 Según Flavio Josefo, el puente cuyos restos se conocen hoy en día como el arco de Wilson unían el monte del Templo con el palacio asmoneo. Cf. Flavio Josefo, Guerra de los judíos II.16.3, en The Works of Flavius Josephus.

17 Solo en el monte del Templo, sin contar las grandes piscinas externas, como las de Betesda y Siloé, por mencionar solo dos de las más grandes, fueron encontradas 37 cisternas. Cf. D. BAHAT, The Illustrated Atlas of Jerusalem, pp. 48-49.

18 E. MAZAR, The Complete Guide to the Temple Mount Excavations. Jerusalén, Shoham Academic Research and Publication, 2012, p. 60. 
Esta hipótesis está basada en el hecho de que Ch. Warren encontró a 14 metros de distancia en dirección oeste -valle Cedrón- de la muralla una sección de una pequeña muralla curvada hacia el sur que muy probablemente contenía una escalera que continuaba en una calle que se unía a esa pequeña puerta doble de la esquina sudeste ${ }^{19}$.

Debemos recordar que la Misná, en el tratado Middot ya citado, refiere que en el lado este del monte del Templo hay una sola puerta. Esta discrepancia podría deberse a que la pequeña puerta doble de la esquina sudeste no se dirigía al espacio sacro superior, sino a las instalaciones subterráneas -conocidas por la tradición como los «establos de Salomón» ${ }^{20}$.

Algunos investigadores han propuesto que la puerta doble -al sur del monte del Templo- podría ser la puerta Hermosa citada en Hch 3,1-2.10. Esto es debido a que las excavaciones arqueológicas han revelado que los pasajes hacia el recinto del Templo que pasaban por debajo de la Stoá estaban decorados con gran finura y sofisticación, incluso algunos de los adornos llegaron hasta el día de hoy ${ }^{21}$. La Misná, en el tratado Middot 2,3, dice que las puertas interiores del Templo -no las de la muralla-, es decir, las que separaban el atrio exterior del edificio mismo del Templo, estaban recubiertas de oro. Todas excepto la puerta de Nicanor, que era de un bronce tan pulido que brillaba como el oro. El tratado Yomá 3,10 recuerda los milagros que se realizaban en la puerta de Nicanor. Flavio Josefo la coloca separando el atrio de los gentiles del atrio de las mujeres, de estilo corintio, y dice que era más hermosa que las que estaban cubiertas de oro ${ }^{22}$. Sin embargo, el tratado Middot 3,6 parece sugerir más bien que se trataba de la puerta más interna, la que separaba el atrio de las mujeres del atrio de Israel, ya que estaba frente a la puerta del santuario. Para que se encontrara un paralítico pidiendo limosna jamás podría haber sido la más cercana al santuario. Tampoco la de la muralla, ya que, como hemos señalado, no se utilizaba. Ritmeyer señala que la tradición de llamar puerta Dorada, es decir, de oro, proviene de este texto de los Hechos de los Apóstoles. El adjetivo ẃpaĩoৎ fue traducido a la Vulgata por aurea, que significa de oro. ${ }^{23}$

19 Cf. L. RITMEYeR, The Quest: Revealing the Temple Mount. Carta Jerusalem. Jerusalén, 2006, p. 107 (Electronic text hypertexted and prepared by OakTree Software, Inc. Version 1.4).

20 Ibid., p. 108.

21 Cf. ibid., p. 74.

22 Cf. Flavio Josefo, Guerra de los judíos V.5.3, en The Works of Flavius Josephus.

${ }^{23}$ Cf. L. \& K. Ritmeyer, Jerusalem. The Temple Mount. Carta Jerusalem. Jerusalén, 2015, p. 116. Cf. J. Murphy-0'Connor, The Holy Bible. An Oxford Archaeological Guide from Earliest Times to 1700. Oxford, University Press, 52008, p. 102. 


\subsection{Período bizantino}

El fabuloso mapa de Madaba, en Jordania, realizado en coloridos mosaicos, muestra la Jerusalén del siglo vi. Aparece allí claramente entre las grandes basílicas, la muralla, con sus puertas, y las calles principales - «cardos»-, la puerta Este del monte del Templo. Los bizantinos habían construido en la puerta una capilla para recordar la curación del paralítico realizada por los apóstoles Pedro y Juan, relatada en Hch 3.

En el siglo VII existe una tradición cristiana que cuenta que el emperador Heraclio, al recuperar la cruz de Cristo, robada por los persas en el año 614 de la basílica del Santo Sepulcro, la trae nuevamente a Jerusalén en el año 630 entrando por la puerta Este, imitando el camino de Cristo hacia el Calvario.

\subsection{Período árabe}

Luego del período temprano árabe (638-1099) comienza el breve período cruzado (1099-1187), durante el cual los cruzados reconstruyen las murallas en los lugares donde estaban destruidas y reconfiguran varias puertas. Tres en el lado norte, una en el lado oeste, tres en lado sur y, finalmente, una habilitada en el lado este, llamada puerta de Josafat, debido a que estaba enfrente del valle de Josafat. Pero esta puerta Este no es la puerta que da al monte del Templo, sino una más al norte, la que actualmente se llama puerta de los Leones ${ }^{24}$.

La puerta Este ya estaba cerrada cuando llegaron los cruzados, pero durante su breve reinado la abrían anualmente en dos ocasiones.

Luego que los cruzados perdieran definitivamente Jerusalén, la puerta fue cerrada definitivamente en el período árabe ayyubid (1187-1250) ${ }^{25}$. Aunque el doble sello de la puerta que podemos ver hoy en día fue realizado por Solimán el Magnífico en 1541, y la tradición islámica llama a cada una de esta puerta doble: puerta de la Misericordia -Bab al-Rahma en árabe- a la del lado sur y puerta del Arrepentimiento -Bab al-Taubah en árabea la del lado norte.

El nombre puerta de la Misericordia proviene de la tradición judeocristiana, que los musulmanes han tomado. De hecho, hoy en día en hebreo

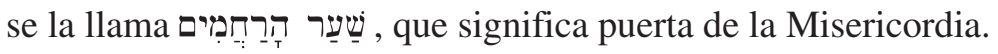

24 Cf. D. BAнат, The Illustrated Atlas of Jerusalem, pp. 92-93.

25 Ibid., p. 79. Cf. L. RITMEYeR, The Quest: Revealing the Temple Mount, p. 9. 


\section{Algunas hipótesis acerca de la actual estructura de la puerta Este}

Debido a la magnificencia de la construcción, algunos dicen que se remonta al tiempo del emperador Justiniano (483-565), ya que fue él quien también construyó y embelleció la ciudad de Jerusalén. Otros sostienen que se remonta al período umayyad ${ }^{26}$ (661-750), debido a que tiene un estilo similar al de otras construcciones de esa época, como por ejemplo la «puerta doble». Otros sostiene que fue construida para un evento particular y de suma importancia, lo cual, unido a lo mencionado anteriormente del retorno de la cruz en el año 629, permite pensar que podría haber sido construida para este propósito por el emperador Heraclio.

Lo cierto es que contiene elementos estéticos del arte bizantino y árabe umayyad. Pero la imposibilidad de realizar estudios arqueológicos impide llegar a una conclusión.

Dentro de la puerta han sobrevivido a las destrucciones dos postes monolíticos: el del lado norte, de 4,5 metros, y el otro de 3,5. Lo interesante que hace notar Ritmeyer ${ }^{27}$ es que el tope superior del poste sur coincide con el nivel de la muralla hasta donde llegan las piedras más antiguas visibles en ella. Sobre ella está la del período árabe temprano, y, sobre esta última, la actual muralla turca ${ }^{28}$. Esta alineación permite hipotetizar que los postes de una antigua puerta fueron construidos al mismo tiempo que la muralla con la cual se alinea; por lo que serían restos de la puerta Este, o bien del primer Templo, o de la reconstrucción de Nehemías. Debido a que Herodes no modificó la línea del recorrido de la muralla este en su ampliación, esta puerta debió de ser utilizada -la original, cuyos restos son los dos postes mencionados- en el siglo I, ya que no hay restos de otra puerta herodiana en la muralla este.

La distancia entre ambos postes marca una apertura de 8,5 metros, lo cual resulta excesivo para una puerta simple; así que seguramente existía un tercer poste central entre ambos.

En 1969 James Fleming ${ }^{29}$, mientras inspeccionaba el área, cayó en una tumba moderna frente a la puerta. Mirando a su alrededor observó los

26 Ritmeyer sostiene esta teoría, pero dice que está sobre los fundamentos de otra más antigua. Cf. L. RITMEYER, The Quest: Revealing the Temple Mount, p. 108.

27 Cf. ibid., p. 109.

28 Sobre la cronología de las piedras de la muralla este, en la sección donde se encuentra la puerta Este, cf. ibid., pp. 174-178.

29 J. FLEMING, «The Undiscovered Gate Beneath Jerusalem's Golden Gate», en Biblical Archaeology Review 9/1 (enero-febrero 1983). 
restos de lo que fue un gran arco. Debido a la profundidad conjeturó que debía tratarse de un arco preherodiano, quizá del período del Primer Templo. Ritmeyer ${ }^{30}$ opina que esto es imposible, debido al alto grado de pendiente que existe en la parte este del monte del Templo, por lo que no habría habido espacio físico para una puerta. Además, según Ritmeyer, las piedras del arco tienen estilo herodiano y están situadas a solo 13 metros de altura de la roca del monte del Templo. Por lo tanto, concluye, ese arco es parte de una rampa o escalera que conducía a la calle que comunicaba con la pequeña puerta doble de la esquina sudeste mencionada anteriormente, similar, aunque más pequeña, a los arcos que sostenían el pasaje ahora llamado arco de Robinson. Debido a que el arco se encuentra intacto y nada hay construido sobre él, excepto que el cementerio musulmán moderno, podrían encontrarse aún los escalones sobre el arco cubiertos por restos de la destrucción del año 70 y el cementerio.

Nuevamente, la imposibilidad de realizar excavaciones arqueológicas en zonas administradas por los musulmanes imposibilitan despejar las dudas.

\section{Importancia de la puerta Este}

Parafraseando la haggadá pascual podríamos preguntarnos: ¿por qué esta puerta es diferente a las otras?, ¿qué la hace tan importante?

La Misná, en el tratado Kelim 17,9, menciona que dos cúbitos -dos medidas, instrumentos para medir de alrededor de 50 centímetros- se encontraban en una cámara sobre puerta Shushan. El término בידָה significa «edificio, palacio, templo», pero, dado el contexto del libro de Nehemías, donde aparece la palabra dos veces $-2,8$ y 7,2 - en relación con la muralla y la puerta Shushan, es claro que se refiere a una habitación que existía en esa puerta. La importancia de estas medidas es que constituían los estándares para cualquier construcción. Y, tratándose de la reconstrucción del Templo, cuyas medidas habían sido dictadas por Dios mismo, la exactitud estaba ligada espiritualmente al cumplimiento de la Palabra de Dios.

Pero tal vez el aspecto más importante de la puerta sea su función ritual, en particular los ritos de la vaca roja y el agua lustral de Nm 19,1-10. Otro tratado de la Misná, Pará, relata todo el rito sacrificial de la vaca roja פָדָדה en hebreo significa justamente vaca-. En el capítulo tercero se descri-

${ }^{30}$ Cf. L. RItmeyer, The Quest: Revealing the Temple Mount, p. 112. 
be que el animal era conducido por un puente de $\operatorname{arcos}^{31}$ desde el Templo hasta el monte de los Olivos, donde era quemada y recolectadas sus cenizas. Estas cenizas, mezcladas con agua de Siloé, eran el «agua lustral» y eran utilizadas para purificar los pecados. Este puente fue costeado por el sumo sacerdote mismo, según especifica la Misná, tratado Shekalim 4,2.

Asimismo, en el día de Yom Kippur -Lv 16- se traía a esta puerta la cabra-Azazel- que era conducida al desierto. El tratado Yomá de la Misná describe con detalle la gran fiesta. Allí, en 1,3, se especifica que en ese solemne día, antes del amanecer, le llevaban al sumo sacerdote, que se encon-

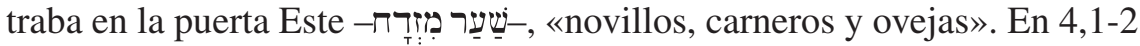
se relata el envío de una de las dos cabras, la dedicada a Azazel, al desierto desde allí.

Si bien la fiesta del Yom Kippur es la más importante y solemne del judaísmo, para poder volver a realizar cualquier sacrificio ritual y restaurar el culto es necesario ante todo la ceremonia de la vaca roja. Ya que con esa agua lustral mencionada se realiza la purificación de los sacerdotes, el altar, el Templo y todos los utensilios. La conexión de este hecho con la puerta Este y su vinculación al monte de los Olivos es hasta el día de hoy de suma importancia por quienes buscan reconstruir un tercer Templo ${ }^{32}$.

La puerta Este es también muy importante en las profecías de Ezequiel. El profeta es llevado en una visión que comienza en el capítulo 40 a una Jerusalén escatológica, celestial. Transcribamos algunos textos de suma importancia para nuestro tema:

El hombre me llevó hacia la puerta que miraba al oriente, y yo vi que la gloria del Dios de Israel venía desde el oriente, con un ruido semejante al de las aguas caudalosas, y la tierra se iluminó con su gloria. Entonces caí con el rostro en tierra. La gloria del Señor entró en la Casa por la puerta que daba al oriente (Ez 43,1-2.4).

El hombre me hizo volver en dirección a la puerta exterior del Santuario, la que miraba hacia el oriente, y esa puerta estaba cerrada. Entonces el Señor

31 «And they would make a causeway from the Temple mount to the Mount of Olives, arches upon arches, an arch directly above each pair, because of the grave in the depths, on which the priest who burns the cow, and the cow, and all those that assist it go forth to the Mount of Olives», Mishná, Pará 3,6. Según el tratado Yoma 6,4, la razón de la construcción del puente no son las tumbas y la posibilidad de quedar impuros pisándolas, sino en recuerdo de los babilonios, que tiraban de sus pelos y llorando gritaban: «Toma nuestros pecados y vete» Sobre la discusión de si era un puente o una rampa en cuanto al término misnaico בבשיש, cf. L. RITMEYER, The Quest: Revealing the Temple Mount, p. 113.

32 Cf. el texto de Rabí Chaim Richman en Rose Guide to Temple, p. 75. 
me dijo: «Esta puerta permanecerá cerrada. No será abierta, y nadie entrará por ella, porque el Señor, el Dios de Israel, ha entrado por ella. Por eso permanecerá cerrada. Solamente el príncipe en ejercicio se sentará allí para comer en la presencia del Señor. Él entrará por el camino del vestíbulo de la puerta y saldrá por ese mismo camino» (Ez 44,1-3).

El hombre me hizo volver a la entrada de la Casa, y vi que salía agua por debajo del umbral de la Casa, en dirección al oriente, porque la fachada de la Casa miraba hacia el oriente. El agua descendía por debajo del costado derecho de la Casa, al sur del altar. Luego me sacó por el camino de la puerta septentrional y me hizo dar la vuelta por un camino exterior, hasta la puerta exterior que miraba hacia el oriente. Allí vi que el agua fluía por el costado derecho (Ez 47,1-2).

La puerta es una puerta santa. Y nadie, excepto el Santo y su príncipe, puede entrar por ella. La gloria del Señor entra por esta puerta santa. Del Templo brota agua hacia el este, sale por la puerta y desciende al valle Cedrón. Por allí continúa su camino hasta el mar Muerto. Ez 47,8-9 destaca que por donde pasa esa agua hay vida, y que incluso al llegar al mar Muerto sus aguas serán saneadas y habrá vida tan abundante que los pescadores acudirán a él.

La tradición cristiana interpreta que Jesús entró por esa puerta el Domingo de Ramos. El relato se encuentra en los cuatro evangelios -Mt 21,117; Mc 11,1-10; Lc 19,28-46 y Jn 12,12-19-, y todos coinciden en que Jesús entró a la ciudad desde el este, específicamente desde Betfagé, en el monte de los Olivos. Así, sentado sobre un asno, cumpliendo la profecía de Zac 9,9, entró al Templo. Dado que la única ${ }^{33}$ puerta para entrar al Templo desde el monte de los Olivos es la puerta Este, Jesús debió de entrar por ella.

Sin embargo, debemos decir que, dada la santidad de la puerta Este descripta anteriormente tanto en las profecías bíblicas como en la Misná, es muy poco probable que Jesús entrara por ella, ya que solo el sumo sacerdote -quizá con los sacerdotes asistentes, no está claro esto último en la Misná- podía utilizar la puerta para las ceremonias de la vaca roja y del Yom Kippur.

Si a esto le sumamos el hecho de que -excepto en Jn, que lo traspone al comienzo del evangelio- inmediatamente después de haber entrado al Templo, Jesús expulsó a los vendedores de animales y a los cambistas de monedas, es muchísimo más probable que entrara por las típicas puertas

33 Descontamos la pequeña puerta doble de la esquina sudeste, de la que ya hemos hablado, dado que estaba dentro de la muralla menor exterior, que salía de la puerta Este, se curvaba hacia el sur y así resguardaba la calle del período asmoneo. 
por las que entraba todo el pueblo, es decir las de Huldá, la doble y triple, que se encuentran en el lado sur de la muralla.

Hay dos indicios arqueológicos que refuerzan esta hipótesis. Primero, esas puertas se utilizaban para el motivo dicho. De hecho, a la derecha de la puerta doble se puede ver hoy día una inmensa miqveh, es decir, una piscina de purificación con capacidad de unos 40.000 metros cúbicos de agua $^{34}$. Rito necesario antes de entrar al Templo, especialmente en época de fiestas -y el Domingo de Ramos está en el contexto de la fiesta de Pascua; justamente por eso Jesús había peregrinado a Jerusalén. El segundo indicio es que en el lado sur, apenas cruzando las puertas de Huldá, se encontraba la Stoá, que es un edificio de pórticos inmenso ${ }^{35}$ de dos pisos y más alto que la muralla, que se utilizaba para reuniones y discusiones. Y en la parte inferior estaban los negocios de venta de animales y de cambio de moneda. Esta actividad, en contacto con monedas impuras -porque justamente debían ser cambiadas, ya que contenían la imagen del emperador, hecho blasfemo para el judaísmo- y con los desperdicios de los animales, que también volvían impuro por su contacto, no podía ser realizada en el Templo en sí mismo. La Stoá fue una ampliación hecha por Herodes hacia el sur del espacio sacro del Templo, por lo que no formaba parte de él, al estar más allá de las medidas del tamaño ordenadas por Dios.

Luego que la puerta fuese sellada por los musulmanes -como ya mencionamos-, primero en el primer período árabe, nuevamente luego de la reapertura de los cruzados y, finalmente, en época turca, la tradición cristiana interpretó que esta puerta está sellada cumpliendo la profecía de Ez 43. Y que, cuando vuelva el Señor -la segunda venida escatológica de Jesucristo-, será reabierta y entrará por ella. Esto se basa en que el libro de los Hechos de los Apóstoles $(1,11)$ dice que, al ser Jesús elevado a los cielos en el monte de los Olivos, unos ángeles se aparecieron a los apóstoles y «les dijeron: "Hombres de Galilea, ¿por qué siguen mirando al cielo? Este Jesús que les ha sido quitado y fue elevado al cielo vendrá de la misma manera que lo han visto partir"». Coincidiendo con la profecía de Ezequiel de que el Señor vendrá del Oriente, desde el punto de vista del Templo: del monte de los Olivos.

Esta idea se entronca con las profecías escatológicas de Jl 4,12-15, que mencionan que el Día del Señor, él mismo se sentará en el valle de Josafat a juzgar

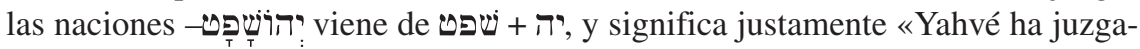
do». Luego lo llama valle de la Decisión. El valle de Josafat es el valle Cedrón,

${ }^{34}$ Cf. E. Mazar, The Complete Guide to the Temple Mount Excavations, p. 61.

35 Ibid., pp. 33-34. 
frente a la puerta Este. Allí Dios tendrá misericordia de Israel, porque «... juzgaré a todas las naciones que han dispersado mi pueblo y repartido mi tierra» $(\mathrm{Jl} 4,1-2)$.

Por tanto, la tradición judía del juicio misericordioso del Dios de Israel es retomada por la tradición cristiana en cuanto a Jesús, el Mesías. Ya que, para «salvar al pueblo de sus pecados», tuvo que entrar en Jerusalén para sufrir en la cruz. Así tuvo misericordia. Y de la misma forma, como ya hemos mencionado, volverá para la consumación escatológica.

\section{Conclusión}

El breve recorrido que hemos realizado por los textos y los monumentos nos permite concluir que la importancia arqueológica, histórica y espiritual de la puerta Este está tan inserta en las tradiciones religiosas que perduran con intensidad hasta el día de hoy.

Especialmente de suma importancia para el judaísmo, por su vinculación con los ritos de la vaca roja, necesaria para la purificación, y del Yom Kippur, la fiesta de expiación de los pecados por excelencia. No lo es menos para el cristianismo, a pesar de que arqueológicamente es muy improbable que Jesús entrara el Domingo de Ramos por esta puerta; el simbolismo espiritual está profunda y fuertemente inserto en la tradición cristiana y el imaginario popular.

Para ambas religiones, toda la zona del este del monte del Templo está ligada a la escatología. Por eso, desde tiempos muy antiguos existen cementerios allí, a la espera del juicio final y la resurrección de los muertos.

En la puerta Este, judíos y cristianos esperan alcanzar la misericordia del Señor. Los judíos, con la venida del Mesías; los cristianos, con su segunda venida escatológica ${ }^{36}$.

Daniel Alejandro Cutri

Jerusalén

danielcutri@gmail.com

${ }^{36}$ Existe una tradición oral que cuenta que la puerta Este fue tapiada y el cementerio musulmán fue construido para que no entrara el Mesías a reconstruir el Templo escatológico. Ya que, de esta forma, al no poder pisar las tumbas y quedar impuro, no podrá entrar al monte del Templo. Sin embargo, las repetidas clausuras de las puertas podrían deberse más bien a la función misma de una muralla y una puerta, que es la de proteger la ciudad de invasiones. La existencia del cementerio es mucho más reciente. Por lo que no podemos concluir si esta tradición tiene fundamento o es un mito popular. 